\title{
Verification of an Approximate Thermodynamic Equation with Application to Study on Arctic Stratospheric Temperature Changes
}

\author{
RENOIANG LIU \\ Collaborative Innovation Center on Forecast and Evaluation of Meteorological Disasters, Key Laboratory of \\ Meteorological Disaster, Ministry of Education, and International Joint Research Laboratory on Climate and \\ Environment Change, and School of Atmospheric Sciences, Nanjing University of Information Science and \\ Technology, Nanjing, China \\ YANYAN FU \\ School of Atmospheric Sciences, Nanjing University of Information Science and Technology, Nanjing, China
}

(Manuscript received 13 April 2018, in final form 2 October 2018)

\begin{abstract}
Temperature changes in the Arctic lower stratosphere on both short- and long-term time scales are critical for changing the magnitude of ozone losses in the Arctic vortex. In this paper, an approximate month-tomonth temperature change equation is constructed and extended to a new form for decade-to-decade changes. Then we provide a verification of these equations and show an example of an application for partitioning between the dynamical and radiative contributions to the Arctic lower-stratospheric temperature decadal changes, as well as the trends, using the European Centre for Medium-Range Weather Forecasts (ECMWF) interim reanalysis (ERA-Interim) data during the period of 1980-99. At $100 \mathrm{hPa}$, the month-tomonth Arctic temperature increment is a small term compared to the dynamical heating and diabatic heating, which are largely canceling terms with maximum magnitudes in November-April and October-March, respectively. However, it is not the case for their decadal changes and the decadal change of the Arctic currentmonth temperature compared to those of the regressed dynamical heating and radiative heating, where the current-month decadal changes and the corresponding trends are approached except in March and a rough agreement exists between these trends and those reported in other studies. The dynamical plus diabatic heating term and the temperature increment, as well as their decadal changes, are roughly balanced during the annual oscillation. However, some departures exist in both cases because of the large deviations or uncertainties of relevant terms and also probably due to the quasigeostrophic approximation and the eddy heat flux approximation of the dynamical heating, and a restricted condition of the eddy heat flux approximation is given at the end.
\end{abstract}

\section{Introduction}

Under quasigeostrophic scaling, the radiative and dynamical heating rates control the zonal-mean stratospheric temperature (e.g., Rosenlof 1995; Ivy et al. 2016). Starting from the quasigeostrophic transformed Eulerian-mean (TEM) equations (Andrews et al. 1987), Newman et al. (2001) first and Hu and Tung (2002) subsequently derived two similar simplified thermodynamic energy equations in integral form. These equations are obtained by taking the area-weighted average over an area from some reference latitude to the pole. However, the dynamical heating correlated with the current zonal-mean temperature or

Corresponding author: Prof. Renqiang Liu,rq_liu@nuist.edu.cn temperature increment is approximated solely by the cumulative eddy heat flux term at that reference latitude [hereafter referred to as the eddy heat flux (EHF) approximation of the dynamical heating]. The derivations are based on the downward control principle (Haynes et al. 1991) or the steady-state assumption of the zonal-mean momentum equation via applying suitable boundary conditions and neglecting the eddy momentum flux forcing term at the reference latitude. The main difference is that Hu and Tung (2002) did not treat the diabatic heating term but Newman et al. (2001) had approximated it as the standard Newtonian cooling approximation (Fels 1982) and argued that the preceding wave driving will have limited impact on the current temperature. While both approximate equations may be applicable to the study on 
the Arctic lower-stratospheric temperature changes, neither has been quantitatively verified.

Through detailed correlation analysis, Newman et al. (2001) showed that the interannual variability of March polar stratospheric temperatures during the period 19792000 is principally related to the tropospheric to lowerstratospheric eddy heat flux observed in January-February. They further used a simple linear fitting to explain, over the averaged annual cycle, the wave-driven and "dynamics free" components of current-month stratospheric temperature and compared the dynamics-free temperature with a radiation model estimate of the temperature in the absence of dynamics. Following their work, Bohlinger et al. (2014) proposed a multivariate linear regression model to separate the dynamical and radiative contributions to the Arctic stratospheric temperature trends. In contrast, Ivy et al. (2016) exploited an offline radiative transfer model to directly evaluate the radiative temperature trends and then estimated the dynamical contribution to the trends as a residual from the total observed.

In this study, using the European Centre for MediumRange Weather Forecasts (ECMWF) interim reanalysis (ERA-Interim) data, we shall provide a verification of one approximate thermodynamic equation, which is in the form of $\mathrm{Hu}$ and Tung (2002) but combined with the integration time scale suggested by Newman et al. (2001), and show an example of an application for partitioning between the dynamical and radiative contributions to the Arctic lower-stratospheric temperature decadal changes as well as the trends. In the discussion, we will derive a restricted condition of the EHF approximation of the dynamical heating. Prior to this, however, main theoretical methods that underpin the calculation and some details about the data employed will be described.

\section{Theoretical considerations}

Following $\mathrm{Hu}$ and Tung (2002), we can derive a dayto-day valid temperature differential equation:

$$
\frac{\partial}{\partial t}\langle\bar{T}\rangle \approx F_{\varphi, z}+\langle\bar{Q}\rangle,
$$

where the overbar refers to a zonal mean, the angle brackets denote the area-weighted average from a reference latitude $\varphi$ to the pole,

$$
F_{\varphi, z}=\left.\frac{\overline{v^{\prime} T^{\prime}} \cos \varphi}{a(1-\sin \varphi)}\right|_{\varphi, z}
$$

is the meridional eddy heat flux term at the latitude $\varphi$ and $\log$ pressure altitude $z, T$ and $Q$ are evaluated at the same altitude $z$, and

$$
\langle\bar{Q}\rangle=\langle\bar{J}\rangle / c_{p}
$$

the diabatic heating term with $J$ the diabatic heating rate per unit mass. All other terms are as defined by Andrews et al. (1987).

Integrating (1) from $t_{1}$ (e.g., 1 January) to $t_{2}$ (e.g., 31 January) gives

$$
\langle\bar{T}\rangle_{t_{2}}-\langle\bar{T}\rangle_{t_{1}}=\int_{t_{1}}^{t_{2}} F_{\varphi, z} d t+\int_{t_{1}}^{t_{2}}\langle\bar{Q}\rangle d t
$$

where the integration time length $t_{2}-t_{1}$ may be approximated as the radiative relaxation time scale $\tau$, which is nearly 30 days in the lower stratosphere as suggested by Newman et al. (2001).

Sliding (4) day by day and summing and averaging, one can construct a month-to-month temperature change equation:

$$
\begin{aligned}
\frac{1}{M} \sum_{i=1}^{M}\langle\bar{T}\rangle_{t_{2}+i d}-\frac{1}{M} \sum_{i=1}^{M}\langle\bar{T}\rangle_{t_{1}+i d}= & \frac{1}{M} \sum_{i=1}^{M} \int_{t_{1}+i d}^{t_{2}+i d} F_{\varphi, z} d t \\
& +\frac{1}{M} \sum_{i=1}^{M} \int_{t_{1}+i d}^{t_{2}+i d}\langle\bar{Q}\rangle d t
\end{aligned}
$$

where $M$ may be set to 30 but not necessarily so and $i d=i \times$ 1 day expresses the time increment of $i(=1,2, \ldots, M)$ days.

For convenience, we rewrite (5) simply as

$$
T_{2}-T_{1}=F+Q,
$$

where $T_{2}, T_{1}, F$, and $Q$ denote corresponding terms in (5); for example,

$$
T_{2}=\frac{1}{M} \sum_{i=1}^{M}\langle\bar{T}\rangle_{t_{2}+i d}
$$

The month whose temperature is $T_{2}$ will be referred to as the current month.

We may further extend (6) to a new form for studying the decade-to-decade temperature changes as

$$
\Delta\left(T_{2}-T_{1}\right)=\Delta(F+Q)
$$

or

$$
\Delta T_{2}-\Delta T_{1}=\Delta F+\Delta Q,
$$

where $\Delta$ represents the difference in decadal-scale means for each term in (5); for example,

$$
\Delta T_{2}={\overline{\frac{1}{M} \sum_{i=1}^{M}\langle\bar{T}\rangle_{t_{2}+i d}}}_{1990 \mathrm{~s}}-{\overline{\frac{1}{M} \sum_{i=1}^{M}\langle\bar{T}\rangle_{t_{2}+i d}}}_{1980 \mathrm{~s}} .
$$


However, note that the decadal changes are generally not the trends. Decadal changes include the lowerfrequency variations incorporated with the trends. When the lower-frequency components are weak, the trends can be approximated by decadal changes.

\section{Data}

Daily averaged meridional wind, temperature, and diabatic heating data at a horizontal resolution of $2.5^{\circ} \times$ $2.5^{\circ}$ from ERA-Interim are used to study the Arctic lower-stratospheric temperature changes (Dee et al. 2011). To simplify the problem and compare results with those reported in other studies, we will look at the period 1980-99, when the global increase in carbon dioxide and the stratospheric ozone depletion are nearly monotonic. Short terms 1982-84 and 1991-93 are excluded since the lower-stratospheric temperatures are strongly affected, respectively, by the eruptions of El Chichón and Mount Pinatubo (Santer et al. 2000). Thus, the data are a little short for investigation of decadal changes as well as the trends. However, we may also avoid detecting a climatic turning point around the 2000s (e.g., Liu et al. 2010). The diabatic heating is calculated from ECMWF model forecasts. Fueglistaler et al. (2009) showed that the diabatic heat budget is better balanced in ERA-Interim and the temperature assimilation increment substantially smaller than in ERA-40. Even so, some errors will remain because of, for example, the use of a zonal-mean, monthly mean ozone climatology in the forecast model for the radiative transfer calculation. The meridional eddy heat flux term in (2) is evaluated at $100 \mathrm{hPa}$, where the flux can represent the upward wave propagation from the troposphere into the stratosphere (Edmon et al. 1980; Hartmann et al. 2000). To reduce uncertainty and after our test, an area-weighted average over $45^{\circ}-75^{\circ} \mathrm{N}$ of the eddy heat flux term is estimated instead of that at the reference latitude of $60^{\circ} \mathrm{N}$, as done in Newman et al. (2001) and Bohlinger et al. (2014).

In principle, we should use some "perfect" dataset, which truly represents the state and variability of the atmosphere, to verify the approximate thermodynamic equation. However, even the observations have their problems. Meanwhile the temporal continuity and spatial coverage of the data have to be considered. So we would prefer to use the reanalysis.

\section{Verification of the approximate temperature change equations}

Figure 1 shows the month-to-month Arctic temperature increment, dynamical heating, diabatic heating, and dynamical plus diabatic heating mean terms during the period 1980-99, calculated at $100 \mathrm{hPa}$ for each month from ERA-Interim according to (5). From Fig. 1a, we see that the month-to-month Arctic temperature increment is a small term (but with a nonzero annual cycle) compared to the dynamical heating and diabatic heating, which are largely canceling terms with maximum magnitudes in November-April and October-March, respectively, and maximum deviations in winter-spring. All the three terms reach their minimum magnitudes and deviations in summer. Figure $1 \mathrm{~b}$ shows that the dynamical plus diabatic heating term and the temperature increment are roughly balanced during the annual oscillation. Their correlation coefficient is 0.67 , which is significant at the $95 \%$ confidence level. However, the dynamical plus diabatic heating term is larger during winter-spring months and slightly smaller during summer-autumn, indicating the dynamical heating and diabatic cooling are dominant, respectively, in different seasons. There may be a concern about the opposite signs of the dynamical plus diabatic heating term and the temperature increment in December and January, although this is not necessarily a situation more serious than that in February, March, and even September regarding the large deviations of each term in the two months (see Fig. 1a). Possible sources of deviations include the interannual variations of each term, observational uncertainties in the Arctic lower-stratospheric ozone and meteorological fields (Randel and Wu 2007; Thompson et al. 2012), effect of the data assimilation method for producing the diabatic heating rates (Fueglistaler et al. 2009; Dee et al. 2011), and the precision and accuracy of the eddy heat flux derived from reanalysis during the maximum wave activity conditions of the Northern Hemisphere winter (Newman and Nash 2000), but an assessment for the relative impact of each deviation factor is beyond the scope of this study. Theoretically, the steady-state assumption of the zonal-mean momentum equation required in the derivation of the EHF approximation is another possible source of error, and a restricted condition of the EHF approximation will be given in the discussion.

Figure 2 shows the decadal changes of the month-tomonth Arctic temperature increment, dynamical heating, diabatic heating, and dynamical plus diabatic heating during the period 1980-99, calculated at $100 \mathrm{hPa}$ for each month from ERA-Interim according to (9). From Fig. 2a, we see that the decadal change of Arctic temperature increment is not a small term compared to those of the dynamical heating and diabatic cooling, which are dominant in winter-spring and summer-fall, respectively (except comparable in April). Figure $2 b$ shows that the decadal changes of the temperature increment and the dynamical plus diabatic heating are roughly balanced year-round. Their correlation coefficient is 0.96 , which is significant at the $99 \%$ confidence level. Some differences 
a)

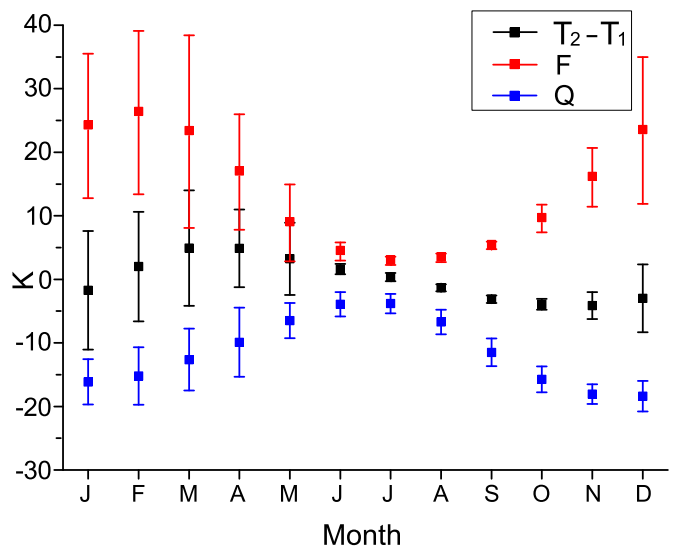

b)

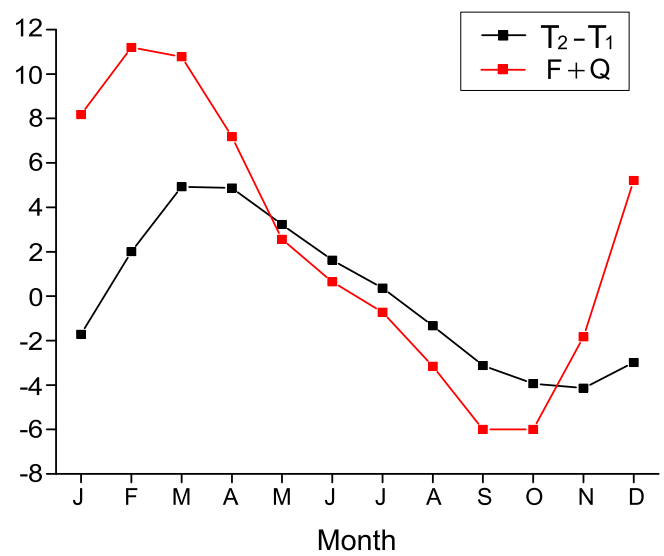

FIG. 1. Month-to-month Arctic $\left(60^{\circ}-90^{\circ} \mathrm{N}\right)$ (a) temperature increment (black), dynamical heating (red), and diabatic heating (blue), and (b) temperature increment (black) and dynamical plus diabatic heating (red) mean terms during the period 1980-99, calculated at $100 \mathrm{hPa}$ by month from the ERA-Interim data based on a simplified thermodynamic energy equation. Vertical bars in (a) show the $2 \sigma$ deviations.

exist in January-April. More homogeneous inconsistency occurs, however, in August-November and seems related to the continuously negative values of diabatic heating change during this time (see Fig. 2a).

\section{An example of application}

There is a robust eddy heat flux-temperature correlation (highest in winter and spring) in the lower stratosphere as noted by Newman et al. (2001) and reproduced by chemistry-climate models (Eyring et al. 2010). The month-to-month temperature change equation [see (5)] indeed gives a balance constraint between the month-tomonth temperature increment and the dynamical plus diabatic heating term. However, it neither shows explicitly the relationship between the current-month temperature and the accumulated wave forcing nor extends such a relationship to decadal or interdecadal time scales. Thus, we may apply a multivariate linear regression to monthly mean temperatures (Bohlinger et al. 2014):

$$
T_{2} \approx \alpha+\beta t+\gamma F
$$

where $T_{2}$ and $F$ are as specifically defined in (6), $t$ is in units of year, and $\alpha, \beta$, and $\gamma$ are the regression parameters, to separate the effects on the decadal-scale temperature changes due to radiative processes, that putatively follow the long-term changes of radiatively active gases, and dynamical processes congruent with changes in the cumulative eddy heat flux. We actually obtain 1) the dynamical component time series by regressing $T_{2}$ onto $F$, 2 ) the residual time series by subtracting point 1 from the current-month temperature time series, 3) the putative radiative component time series by a least squares fitting to point 2, and hence 4) the monthly decadal changes and the corresponding trends (Thompson et al. 2000; Bohlinger et al. 2014).

Figure 3 shows the decadal changes of the Arctic current-month temperature, cumulative dynamical heating, and putative radiative heating during the period 1980-99, calculated at $100 \mathrm{hPa}$ for each month from ERA-Interim according to the multivariate linear regression in (11). From Fig. 3, we see that, as in Fig. 2a, the decadal change of the Arctic current-month temperature is not a small term compared to those of the regressed dynamical heating and radiative heating, which are dominant in winter and spring-summer, respectively (except comparable in January). However, evident differences appear in Figs. 2a and 3. In particular, the decadal changes of month-to-month Arctic temperature increment and current-month temperature have opposite signs in April, those of the dynamical heating terms have a largemagnitude difference in March, and those of the diabatic heating terms are of opposite signs in the whole spring. The strongly negative radiative heating changes shown in Fig. $3 c$ probably associate with the peak ozone depletion observed in the Arctic springtime (Randel and Wu 2007; Ivy et al. 2016), while their large gradient from March to May could contribute, illustratively, to the strongly positive diabatic changes shown in Fig. 2a. Also plotted in Fig. 3 alongside the decadal changes are the corresponding monthly trends. The signs and magnitudes of two changes are approached except in March, suggesting that the trend part is the main component of the decadal change and hence statistically meaningful. Furthermore, there is a rough agreement in the seasonal cycles and magnitudes 
a)
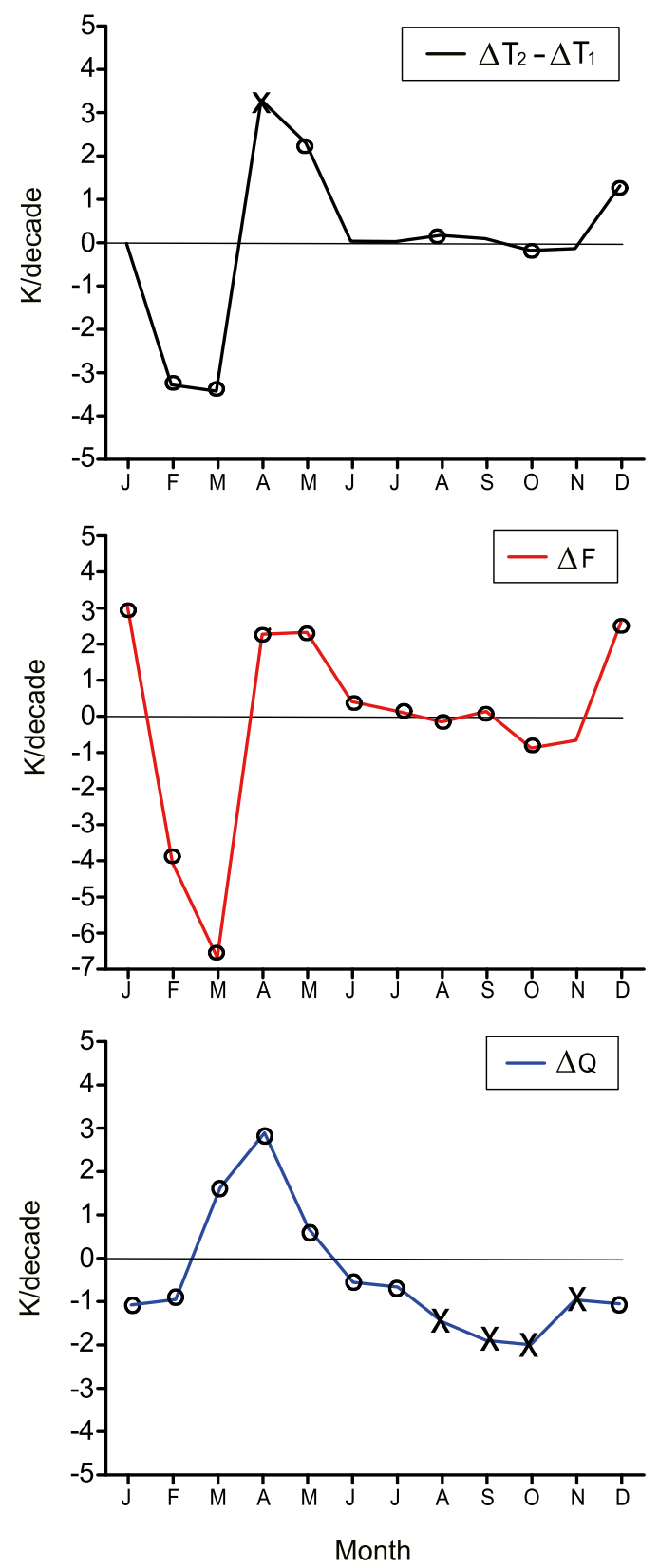

b)

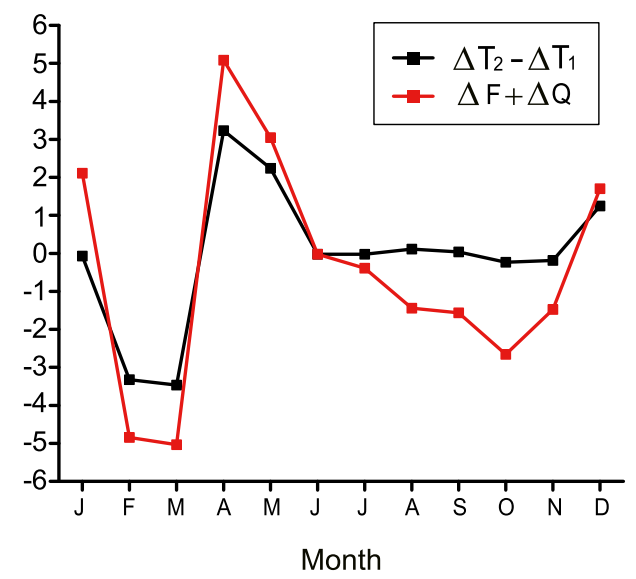

FIG. 2. Decadal changes (1990s minus 1980s) of the month-to-month Arctic $\left(60^{\circ}-90^{\circ} \mathrm{N}\right)$ (a) temperature increment (black), (a) dynamical heating (red), and (a) diabatic heating (blue), and (b) temperature increment (black) and dynamical plus diabatic heating (red) during the period $1980-99$, calculated at $100 \mathrm{hPa}$ by month from the ERA-Interim data based on a simplified thermodynamic energy equation. Symbols in (a) indicate decadal changes that are significantly different from zero at the $1 \sigma$ (circles) or $2 \sigma$ levels (crosses). between the trends presented here and those reported in Bohlinger et al. (2014) and Ivy et al. (2016); for example, the dynamical contribution to the temperature trend is dominant in winter, while the radiative contribution is dominant in summer, if neglecting the differences in heights and lengths of the respective analyzed data. However, the radiative temperature trends in winter and spring differ significantly between these studies, possibly because of the exclusion of water vapor in their direct radiative calculation (Ivy et al. 2016) and, on the other hand, the removal of the volcanic effects in our putative analysis (Santer et al. 2000). Notwithstanding the present discrepancies, all studies have provided independent thoughts and complementary approaches for the attribution of decadalscale Arctic stratospheric temperature changes.

\section{Conclusions and discussion}

The temperature changes in the Arctic lower stratosphere on both short- and long-term time scales are 
a)

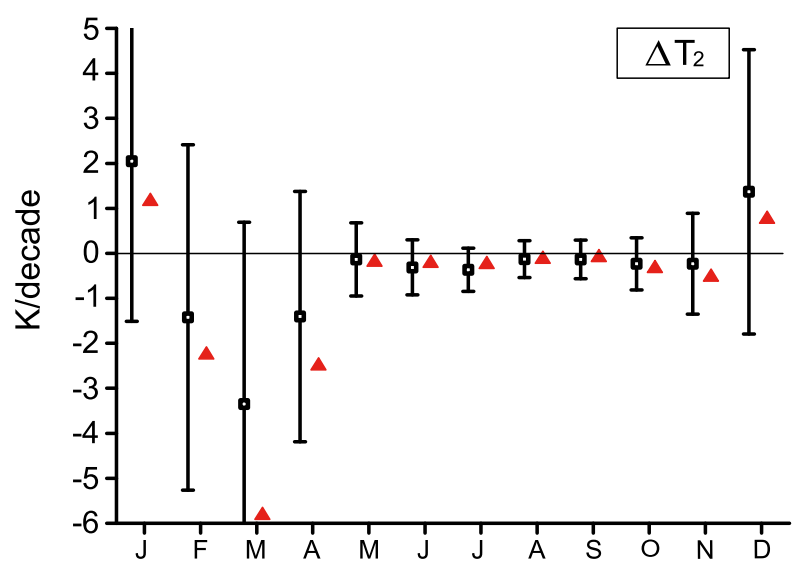

b)

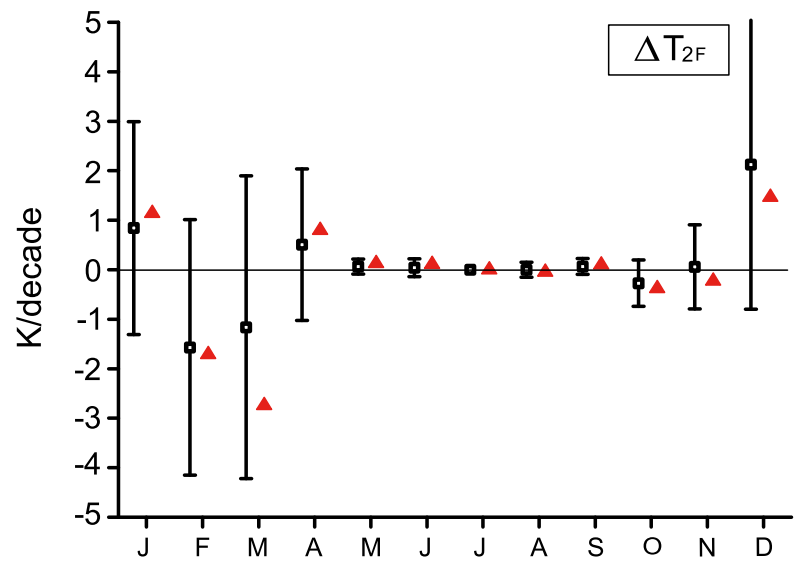

c)

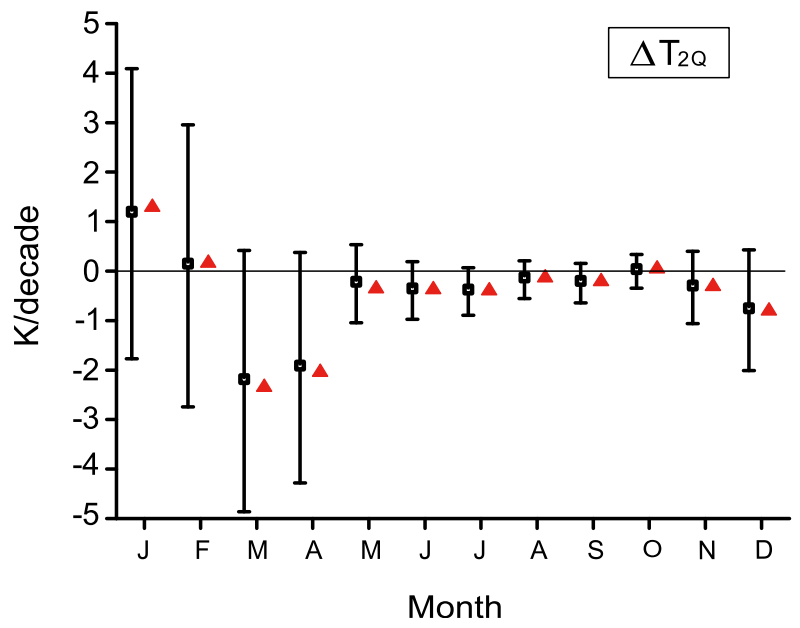

FIG. 3. Decadal changes (1990s minus 1980s; red) and trends (black) of the Arctic $\left(60^{\circ}-90^{\circ} \mathrm{N}\right)$ (a) current-month temperature, (b) cumulative dynamical heating, and (c) putative radiative heating during the period 1980-99, calculated at $100 \mathrm{hPa}$ by month from the ERA-Interim data based on a multivariate linear regression. Vertical bars show the $2 \sigma$ trend uncertainty. critical for changing the magnitude of ozone losses in the Arctic polar vortex. In this study, we first propose a timesliding calculation scheme of one approximate thermodynamic equation that is in the form of $\mathrm{Hu}$ and Tung (2002) but combined with the integration time scale suggested by Newman et al. (2001) and hence construct an approximate month-to-month temperature change equation based on the daily data and extend it to a new form for decade-to-decade changes. We then provide a verification of these equations and show an example of application for partitioning between the dynamical and radiative contributions to the Arctic lower-stratospheric temperature decadal changes as well as the trends, using ERA-Interim data during the period 1980-99.

It is seen that, at $100 \mathrm{hPa}$, the month-to-month Arctic temperature increment is a small term compared to the dynamical heating and diabatic heating, which are largely canceling terms with maximum magnitudes in November-April and October-March, respectively, and maximum deviations in winter-spring. However, the decadal change of Arctic temperature increment is not a small term compared to those of the dynamical heating and diabatic cooling, which are, respectively, dominant in winter-spring and summer-fall. Again, the decadal change of the Arctic current-month temperature is not a small term compared to those of the regressed dynamical heating and radiative heating, which are dominant in winter and spring-summer, respectively. The signs and magnitudes of the monthly decadal changes and the corresponding trends are approached except in March. Furthermore, there is a rough agreement in the seasonal cycles and magnitudes between the trends presented in this study and those reported in Bohlinger et al. (2014) and Ivy et al. (2016); for example, the dynamical contribution to the temperature trend is dominant in winter, while the radiative contribution is dominant in summer.

The dynamical plus diabatic heating term and the temperature increment are roughly balanced during the annual oscillation. Meanwhile, the decadal changes of them are roughly balanced year-round. However, some departures exist in both cases because of the large deviations or uncertainties that may arise from interannual variations and data errors of relevant terms, and also probably due to the quasigeostrophic approximation and the EHF approximation of the dynamical heating. It means that, on the one hand, the simplified thermodynamic energy equation can be used to diagnose the Arctic lower-stratospheric temperature changes on both shortand long-term time scales, and on the other hand, one should be careful in analyzing and explaining the results obtained on the basis of this special simplification.

The steady-state assumption of the zonal-mean momentum equation required in the derivation of the EHF 
approximation within the TEM framework is, intrinsically, a possible source of error. However, if directly starting from the quasigeostrophic Eulerian-mean (EM) thermodynamic energy equation (Andrews et al. 1987, p. 124) and taking the same area-weighted average from a reference latitude $\varphi$ to the pole, we can obtain

$$
\frac{\partial}{\partial t}\langle\bar{T}\rangle=F_{\varphi, z}+\langle\bar{Q}\rangle-N^{2} H R^{-1}\langle\bar{w}\rangle,
$$

where $N$ is the log pressure buoyancy frequency, $H$ the mean scale height, $R$ the gas constant for dry air, $\bar{w}$ the vertical component of the EM circulation, and all other terms are as defined in (1). So whether the EM heating term $-N^{2} H R^{-1}\langle\bar{w}\rangle$ can be neglected in (12) should be a restricted condition of the EHF approximation of the dynamical heating. This condition will be studied in future work to determine the reasonable range of the EHF approximation with regard to the reference latitude, pressure height, season, and integration time scale and hence disclose more application aspects of the simplified thermodynamic energy equation. An assessment for the relative impact of each deviation factor is also needed to give an accurate explanation for sources of some large departures and limits of the approximate temperature change equation. This is particularly pertinent to the attribution of decadal-scale Arctic stratospheric temperature changes due to the internal dynamics variability and anthropogenic climate forcings. Obviously, verifications and comparisons using more and different datasets are required.

Acknowledgments. We thank two anonymous reviewers for their very valuable comments and suggestions. This work was supported by the National Natural Science Foundation of China (Grants 41575040 and 91537213) and the Priority Academic Program Development of Jiangsu Higher Education Institutions (PAPD). The provisions of online data by ECMWF are gratefully acknowledged.

\section{REFERENCES}

Andrews, D. G., J. R. Holton, and C. B. Leovy, 1987: Middle Atmosphere Dynamics. Academic Press, 489 pp.

Bohlinger, P., B.-M. Sinnhuber, R. Ruhnke, and O. Kirner, 2014: Radiative and dynamical contributions to past and future Arctic stratospheric temperature trends. Atmos. Chem. Phys., 14, 1679-1688, https://doi.org/10.5194/acp-14-1679-2014.

Dee, D. P., and Coauthors, 2011: The ERA-Interim reanalysis: Configuration and performance of the data assimilation system. Quart. J. Roy. Meteor. Soc., 137, 553-597, https://doi.org/10.1002/qj.828.

Edmon, H. J., Jr., B. J. Hoskins, and M. E. McIntyre, 1980: EliassenPalm cross sections for the troposphere. J. Atmos. Sci., 37, 2600-2616, https://doi.org/10.1175/1520-0469(1980)037<2600: EPCSFT>2.0.CO;2; Corrigendum, 38, 1115, https://doi.org/ 10.1175/1520-0469(1981)038<1115: > 2.0.CO;2.
Eyring, V., T. G. Shepherd, and D. W. Waugh, Eds., 2010: SPARC report on the evaluation of chemistry climate models. Stratospheric Processes and Their Role in Climate Rep. 5, WMO/TD-1526, 426 pp., https://www.sparc-climate. org/publications/sparc-reports/sparc-report-no-5/.

Fels, S. B., 1982: A parameterization of scale-dependent radiative damping rates in the middle atmosphere. J. Atmos. Sci., 39, 1141-1152, https://doi.org/10.1175/1520-0469(1982)039<1141: APOSDR $>2.0 . \mathrm{CO} ; 2$.

Fueglistaler, S., B. Legras, A. Beljaars, J.-J. Morcrette, A. Simmons, A. M. Tompkins, and S. Uppala, 2009: The diabatic heat budget of the upper troposphere and lower/mid stratosphere in ECMWF reanalyses. Quart. J. Roy. Meteor. Soc., 135, 21-37, https://doi.org/10.1002/qj.361.

Hartmann, D. L., J. M. Wallace, V. Limpasuvan, D. W. J. Thompson, and J. R. Holton, 2000: Can ozone depletion and global warming interact to produce rapid climate change? Proc. Natl. Acad. Sci. USA, 97, 1412-1417, https://doi.org/10.1073/pnas.97.4.1412.

Haynes, P. H., C. J. Marks, M. E. McIntyre, T. G. Shepherd, and K. P. Shine, 1991: On the "downward control" of extratropical diabatic circulations by eddy-induced mean zonal forces. $J$. Atmos. Sci., 48, 651-678, https://doi.org/10.1175/1520-0469(1991) 048<0651:OTCOED $>2.0 . C O ; 2$.

$\mathrm{Hu}$, Y., and K. K. Tung, 2002: Interannual and decadal variations of planetary wave activity, stratospheric cooling, and Northern Hemisphere annular mode. J. Climate, 15, 1659-1673, https:// doi.org/10.1175/1520-0442(2002)015<1659:IADVOP>2.0.CO;2.

Ivy, D. J., S. Solomon, and H. E. Rieder, 2016: Radiative and dynamical influences on polar stratospheric temperature trends. J. Climate, 29, 4927-4938, https://doi.org/10.1175/JCLI-D-150503.1.

Liu, R. Q., C. Jacobi, P. Hoffmann, G. Stober, and E. G. Merzlyakov, 2010: A piecewise linear model for detecting climatic trends and their structural changes with application to mesosphere/lower thermosphere winds over Collm, Germany. J. Geophys. Res., 115, D22105, https://doi.org/10.1029/2010JD014080.

Newman, P. A., and E. R. Nash, 2000: Quantifying the wave driving of the stratosphere. J. Geophys. Res., 105, 12 485-12497, https://doi.org/10.1029/1999JD901191.

,,- and J. E. Rosenfield, 2001: What controls the temperature of the Arctic stratosphere during the spring? J. Geophys. Res., 106, 19 999-20 010, https://doi.org/10.1029/ 2000JD000061.

Randel, W. J., and F. Wu, 2007: A stratospheric ozone profile data set for 1979-2005: Variability, trends, and comparisons with column ozone data. J. Geophys. Res., 112, D06313, https://doi.org/10.1029/2006JD007339.

Rosenlof, K. H., 1995: Seasonal cycle of the residual mean meridional circulation in the stratosphere. J. Geophys. Res., 100, 5173-5191, https://doi.org/10.1029/94JD03122.

Santer, B. D., T. M. L. Wigley, J. S. Boyle, D. J. Gaffen, J. J. Hnilo, D. Nychka, D. E. Parker, and K. E. Taylor, 2000: Statistical significance of trends and trend differences in layer-average atmospheric temperature time series. J. Geophys. Res., 105, 7337-7356, https://doi.org/10.1029/1999JD901105.

Thompson, D. W. J., J. M. Wallace, and G. C. Hegerl, 2000: Annular modes in the extratropical circulation. Part II: Trends. J. Climate, 13, 1018-1036, https://doi.org/10.1175/1520-0442(2000)013<1018: AMITEC $>2.0 . \mathrm{CO} ; 2$.

, and Coauthors, 2012: The mystery of recent stratospheric temperature trends. Nature, 491, 692-697, https://doi.org/ 10.1038/nature11579. 